\title{
On the size of the winning set in the presence of interest groups
}

\author{
Vjollca Sadiraj, Jan Tuinstra ${ }^{\dagger}$ and Frans van Winden ${ }^{\ddagger}$
}

March 2005

\begin{abstract}
Interest groups are introduced in a spatial model of electoral competition between two political parties. We show that the presence of these interest groups increases the winning set, which is the set of policy platforms for the challenger that will defeat the incumbent. Therefore interest groups enhance the probability of the challenger winning the election.
\end{abstract}

Keywords: Spatial voting models, electoral competition, winning set, interest groups.

JEL classification code: D71; D72.

\section{Introduction}

Special interest groups play an important role in political decision making (Richardson, 1994, Potters and Sloof, 1996). Over the last two decades, a substantial number of theoretical studies have appeared trying to explain the influence of interest groups through campaign contributions, strategic information transmission, and the like (for surveys, see Austen-Smith 1994, Grossman and Helpman, 2001, van Winden, 2003). However, thus far the dynamics of the development of special interest groups and the consequences of membership for the political preferences and voting behavior of voters have been neglected. In this paper we argue that this is an important shortcoming of the present state of the art.

\footnotetext{
*Economic Science Laboratory, University of Arizona, Tucson, USA, vsadiraj@econlab.arizona.edu.

${ }^{\dagger}$ Department of Quantitative Economics and CeNDEF, University of Amsterdam, Amsterdam, The Netherlands, j.tuinstra@uva.nl. This research has been supported by the Neterlands Organisation for Scientific Research (NWO) under a MaGW-Pionier grant.

${ }^{\ddagger}$ Department of Economics and CREED, University of Amsterdam, Amsterdam, The Netherlands, f.a.a.m.vanwinden@uva.nl.
} 
An important role for special interest groups lies in coordinating voting behavior of their members. ${ }^{1,2}$ Since interest groups typically focus on one particular issue this implies that members of such an interest group have to, to a certain extent, disregard their political stances on the other issues. Voters are assumed to be willing to do so in order to exert more influence on the election outcome. Moreover, there is plenty of evidence that group membership in general affects preferences. An important psychological mechanism at work here is identification. Social psychological experiments have shown that even minimal groups, defined by an arbitrary label like yellow or blue and with random assignment of individuals, affect behavior (Brewer, 1979, Tajfel and Turner, 1986). Members derive extra utility from behaving in line with the perceived group interest, even if social sanctions on deviant behavior are absent. In fact, the psychological theory of cognitive dissonance predicts that already the decision to join a group will tend to make the issues represented by that group more attractive, relative to those of the groups that are not chosen (Festinger and Aronson, 1968). Akerlof and Kranton (2000) have shown for various types of economic interactions that taking account of the phenomenon of identification - that is, incorporating identity into the utility function - substantively changes the conclusions of previous economic analyses. In this paper, we apply a similar reasoning to investigate the consequences of identification in a political economic context, using a standard spatial model of electoral competition (see e.g. Enelow and Hinich, 1984). More specifically, our research question concerns the differential impact of the presence of interest groups on electoral competition if voters identify with the issue represented by the interest group of their choice. Making the usual assumption of (weighted) Euclidean distance preferences in the absence of interest groups, essentially, identification in this context implies that the political preferences of voters become more lexicographic. The reason is the extra weight or priority attached to the issue represented by the group they are a member of. Together with the assumption that interest group membership is positively correlated with the level of dissatisfaction with the incumbent's policy, coordination and identification produce our main result which relates to the winning set, i.e. the set of policy platforms with which a challenger can defeat a given incumbent's policy position. Assuming for simplicity a two-dimensional issue space and a uniform distribution of the voters ideal points over this space, we are able to show that the introduction of interest groups in this environment typically increases the winning set. This result is particularly important if one takes into account that in practice political parties do not have complete information about voting behavior, urging them to use instruments like polls for acquiring information. Our result then suggests that in the presence of interest groups, it will be easier for the challenger to find a position defeating the incumbent. Or, put differently, the challenger's probability of winning the election will be enhanced by interest groups.

The organization of this paper is as follows. Section 2 presents the spatial compe-

\footnotetext{
${ }^{1}$ For an empirical illustration, see Chong (1991, p.236): "Black organizations and institutions helped to coordinate the preferences and actions of those who supported the civil rights movement."

${ }^{2}$ Membership of an interest group should be interpreted loosely here. It can refer to a registered membership or, alternatively, to an implicit supportive attitude towards the interest group's objectives. In this paper we do not distinguish between these two.
} 
tition model with interest groups and our main result. The proof of the main result is outlined in Section 3, whereas the formal proofs of the results used there are relegated to the Appendix. Section 4 concludes with a further discussion of relevance and implications.

\section{Spatial competition and interest groups}

Two political parties, an incumbent and a challenger, are assumed to compete for votes by selecting certain policy platforms. Platforms are represented as points in an issue space. We take this issue space to be continuous and equal to $\mathcal{X}=B(O, K) \subset$ $\mathbb{R}^{2}$, that is, political parties can choose platforms on two dimensions from an open ball with center at the origin $O=(0,0)$ and radius $K>0 .{ }^{3}$ There is a continuum of voters where each voter $j$ is characterized by an ideal point $x^{j} \in \mathcal{X}$ and an issue weight vector or profile $s^{j} \in \mathcal{S} \times \mathcal{S}$ where $\mathcal{S}=\left\{s^{1}, s^{2}, \ldots, s^{r}\right\}$, with $\underline{s}=s^{1}<s^{2}<\ldots<s^{r}=\bar{s}$. Let ${ }^{4}$ $\underline{s}>0$ and, without loss of generality, $\bar{s}=1$. Suppose voters' ideal points are uniformly distributed over $\mathcal{X}$ and voters' weights are independently and identically distributed (and also independently from voters' ideal points) according to some distribution on $\mathcal{S}{ }^{5} \quad$ A voter's utility with respect to a certain policy outcome $y \in \mathcal{X}$ is given by the negative of the (weighted) Euclidean distance between this policy outcome and the voter's ideal point. Formally, utility for voter $j$ of policy outcome $y$ is given by ${ }^{6}$

$$
u^{j}(y)=-\left\|x^{j}-y\right\|_{s^{j}}^{2},
$$

where $\left\|x^{j}-y\right\|_{s^{j}}^{2} \equiv s_{1}^{j}\left(x_{1}^{j}-y_{1}\right)^{2}+s_{2}^{j}\left(x_{2}^{j}-y_{2}\right)^{2}$.

Let $y$ be the given position of the incumbent. ${ }^{7}$ In the absence of interest groups voters will vote for that candidate whose position will give him or her the highest utility according to (1). In case of a tie the voter will randomize, with equal probability, between the candidates. Now define by $W(y) \subset \mathcal{X}$ the set of policy platforms $z$ that are expected to attract (strictly) more votes than the incumbent's platform $y$. Clearly, the challenger's objective is to select a policy platform from $W(y)$. Notice that by definition $y \notin W(y)$ since a voter is indifferent between a challenger and the incumbent with identical policy platforms. An equilibrium point of the electoral competition game is defined as a platform $y^{*}$ for which the winning set is empty, i.e. $W\left(y^{*}\right)=\emptyset$. The uniform distribution of voters' ideal points over the issue space $\mathcal{X}$

\footnotetext{
${ }^{3}$ We will return to this assumption in Section 4.

${ }^{4}$ As will become clear when we introduce interest groups, voters with weight 0 on one of the issues do not change their voting behavior when these interest groups enter the scene. Taking $\underline{s}>0$ is therefore an innocuous assumption. Moreover, the alternative assumption that strengths are continuously distributed on $[\underline{s}, \bar{s}]$ would lead to the same results.

${ }^{5}$ That is, for all $s, t \in \mathcal{S}$ and all $v, w \in \mathcal{X}$ we assume that $\operatorname{Pr}\left\{s^{j}=(s, t)\right\}=$ $\operatorname{Pr}\left\{s_{1}^{j}=s\right\} \operatorname{Pr}\left\{s_{2}^{j}=t\right\}$ and $\operatorname{Pr}\left\{s^{j}=(s, t) \mid x^{j}=v\right\}=\operatorname{Pr}\left\{s^{j}=(s, t) \mid x^{j}=w\right\}$.

${ }^{6}$ Notice that, following Enelow and Hinich (1984), we assume that preferences are separable. Our formulation implies that the indifference curves are ellipses with horizontal and vertical axes.

${ }^{7} \mathrm{It}$ is realistic to assume that the challenger is more flexible in choosing a platform, since $(\mathrm{s})$ he cannot be held responsible for the existing policy (see e.g. Kramer, 1977, and Kollman, Miller, and Page, 1992).
} 
implies that in our framework such an equilibrium point will indeed exist (cf. Plott, 1967) and is in fact given by the origin (this statement will be made more precise in Proposition 2 in Section 3). By $W(y ; s)$ we will denote the set of policy platforms defeating the incumbent's platform $y$ in the special case where all voters have weight vector $s$.

The next step is to incorporate interest groups in the model. We model a special interest group as being interested in the policy outcome with respect to only one of the two issues, say the first issue. For every position on the first issue voters can join an interest group. The same holds for every position on the second issue. For expositional reasons we are going to investigate the situation where all voters join an interest group. We follow the modelling approach set out in Sadiraj, Tuinstra and van Winden (2004). Before we describe how voting behavior is coordinated by the interest groups let us first determine how individual voters decide which interest group to join. An individual voter with ideal position $x$ is a potential member of two interest groups, the interest group on the first issue located at position $x_{1}$ and the interest group on the second issue located at position $x_{2}$. Which interest group this voter joins depends upon the incumbent's position and the weights the voter attaches to the two issues. We assume that voters are more inclined to join an interest group on a particular issue the more distant the incumbent's policy position is from their own position on that issue. This assumption is supported by evidence on the importance of discontent and frustration for collective action (see e.g. Kernell, 1977, Lau, 1982, Chong, 1991, Romer, 1996, Javeline, 2003). ${ }^{8}$ Of course, the weight a voter attaches to the issues also comes into play. Hence we assume that, given the incumbent policy platform $y$, voter $j$ decides to join the interest group on the issue $i$ with the largest value of $s_{i}^{j}\left(x_{i}^{j}-y_{i}\right)^{2}$, where $i \in\{1,2\}$. In this way the population of voters is divided over the different interest groups. Now consider the interest group located at position $x_{1}$ on the first issue. Identification with the interest group's stance (see the Introduction) stimulates its members, who take the same position on the first issue, to vote for that political candidate which is closest to the interest group on this issue. Therefore, if $y$ and $z$ are the policy platforms of the two political parties, then a member $j$ of this interest group is taken to vote for the first party according to the following decision rule

$$
\text { voter } j \text { votes for } y \text { if }\left\{\begin{array}{l}
\left|y_{1}-x_{1}\right|<\left|z_{1}-x_{1}\right| \\
\left|y_{1}-x_{1}\right|=\left|z_{1}-x_{1}\right|
\end{array} \text { and }\left|y_{2}-x_{2}^{j}\right|<\left|z_{2}-x_{2}^{j}\right|\right.
$$

and similarly for the second party. Naturally, we will assume that the voter votes with probability $\frac{1}{2}$ for either policy platform if $\left|y-x^{j}\right|=\left|z-x^{j}\right|$. A similar decision rule holds for members of interest groups on the other issue. The introduction of interest groups thus induces a change in the structure of voter preferences from weighted Euclidean distance to lexicographic preferences. Similar to $W(y)$ we denote by $W^{I}(y)$ the set of policy platforms defeating the incumbent's platform $y$ when interest groups

${ }^{8}$ For a model of interest group formation using a similar approach but applied to taxation, see Sadiraj, Tuinstra, and van Winden (2005). 
are present. By $W^{I}(y)$ we will denote the set of policy platforms defeating the incumbent's platform $y$ when interest groups are present.

The aim of this paper is to investigate the consequences of the introduction of interest groups for the winning sets given an incumbent's position $y$, i.e. the areas of the winning sets denoted by $|W(y)|$ and $\left|W^{I}(y)\right|$. Our main result is

Theorem 1 Let $\underline{s}>0$ be given. Denote

$$
\Psi(\underline{s})=\min \left\{\frac{2(\varphi(\underline{s}))^{\frac{3}{2}}}{1+(\varphi(\underline{s}))^{3}}, \frac{1}{\sqrt{1+4 \sqrt{\underline{s}} \varphi(\underline{s})}}\right\},
$$

where $\varphi(\underline{s})=1+\frac{1}{s}$. Then for all $y \in B(O, \Psi(\underline{s}) K) \backslash\{O\}$ the area of the winning set increases in the presence of interest groups.

This result shows that for all incumbent positions $y$ within a prespecified circle within the issue space, the winning set increases in the presence of the interest groups. Note that this circle is shrinking as $\underline{s}$ approaches 0 and that the ray of this circle goes to $\frac{1}{3} K$ as $\underline{s}$ approaches 1 . For an intermediate value of $\underline{s}=\frac{1}{2}$, we have $\Psi\left(\frac{1}{2}\right) K=(1+6 \sqrt{2})^{-\frac{1}{2}} K \approx 0.325 K$, which is rather close to $\frac{1}{3} K$ already. Notice that if the incumbent's position falls outside the region specified by Theorem 1, i.e. the incumbent platform is at least a distance $\Psi(\underline{s}) K$ away from the origin, which is the equilibrium point, the winning set for the challenger is relatively large anyway, whether interest groups are present or not.

\section{Outline of the proof of the Theorem}

In this section we will outline the proof of Theorem 1 in a number of intuitive steps. Formal and rigorous proofs of these different steps are relegated to the appendix. It will be convenient to define, for given $v, w \in \mathbb{R}^{2}$ and $c \in \mathbb{R}_{+}^{2}$

$$
\mathcal{E}_{c}(v, w)=\left\{x \in \mathbb{R}^{2}:\|x-v\|_{c}^{2}<\|w-v\|_{c}^{2}\right\} .
$$

Hence $\mathcal{E}_{c}(v, w)$ contains all the points inside an ellipse, which is centered at the point $v$, and going through $w$. The area of this ellipse equals $\pi \frac{\|w-v\|_{c}^{2}}{\sqrt{c_{1} c_{2}}}$.

First we consider the benchmark model, i.e. electoral competition between political parties in the absence of interest groups. We have the following result.

Proposition 2 Assume voters' ideal positions are independently (across issues and across voters) drawn from the uniform distribution on $\mathcal{X}$ and that voters' weights on each issue are independently drawn from a distribution on $\mathcal{S}$ and are uncorrelated with each other and with the ideal positions. Let $y$ be the platform of the incumbent and let $C=\{(\underline{s}, 1),(1, \underline{s})\}$. Then

1. $W(O)=\emptyset$,

2. $W(y ; s)=\mathcal{E}_{s}(O, y)$, 

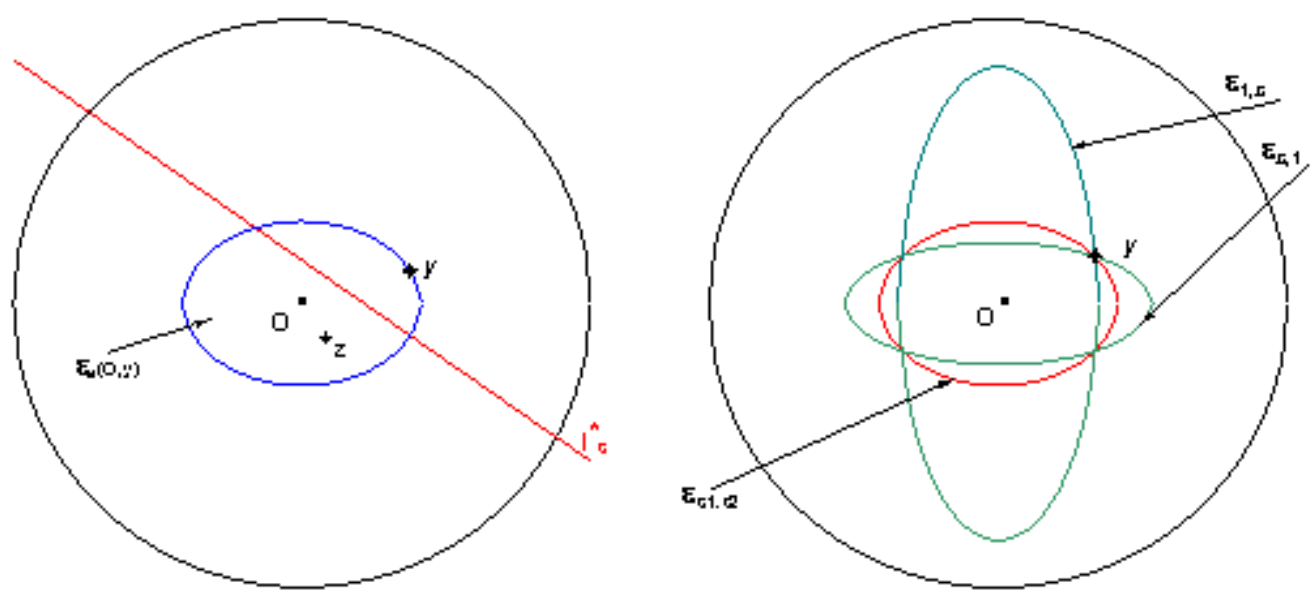

Figure 1: Construction of the winning set for the benchmark model. Left panel shows how $W(y ; s)$ can be constructed (part 2 of Proposition 2), the right panel illustrates part 3 of Proposition 2.

3. $\forall y \in \mathcal{X} \backslash\{O\}, W(y) \subset \bigcup_{c \in C} \mathcal{E}_{c}(O, y)$ and $|W(y)| \leq \pi\|y\|^{2} \varphi(\underline{s}) \sqrt{s}$.

This result is illustrated in Figure 1. Observe that any line through the origin $O$ divides the issue space $\mathcal{X}$ in two subspaces which are equally large. Therefore, since ideal points are symmetrically (and uniformly) distributed over the issue space, no other platform will be able to defeat the origin (the origin here corresponds to the position of the generalized median voter, see Hoyer and Mayer, 1974). Now consider an arbitrary position $z$ in $\mathcal{E}_{s}(O, y)$ (see the left panel of Figure 1). The line $l_{s}^{*}$ presents all positions $x$ such that all voters with weight vector $s$ and ideal point $x$ are indifferent between $z$ and $y$. Therefore all voters with weight vector $s$ and an ideal point below $l_{s}^{*}$ will vote for $z$ and it is then easily seen that the majority of voters with that profile will indeed vote for $z$ (basically, since voters with ideal point $O$ will vote for $z)$. This argument holds for any element of $\mathcal{E}_{s}(O, y)$ and therefore $W(y ; s)=\mathcal{E}_{s}(O, y)$. Moreover, for all $s \in \mathcal{S}^{2}$ we have $\mathcal{E}_{s}(O, y) \subset \bigcup_{c \in C} \mathcal{E}_{c}(O, y)$ (see the right panel of Figure 1), which implies $W(y) \subset \bigcup_{c \in C} \mathcal{E}_{c}(O, y)$. With some tedious but straightforward computations one can then derive the upper bound from part 3 of Proposition 2.

Let us now turn to the model with interest groups. We will derive a lower bound for the size of the winning set in the model with interest groups in a number of steps. The results are driven by the fact that decisions of interest group members are determined by lexicographic preferences, instead of by weighted Euclidean distance. Consider all voters with a certain weight vector $s \in \mathcal{S}^{2}$. Consider a given position $y$ of the incumbent. From now on we will, without loss of generality, assume $y_{1} \geq 0$ 


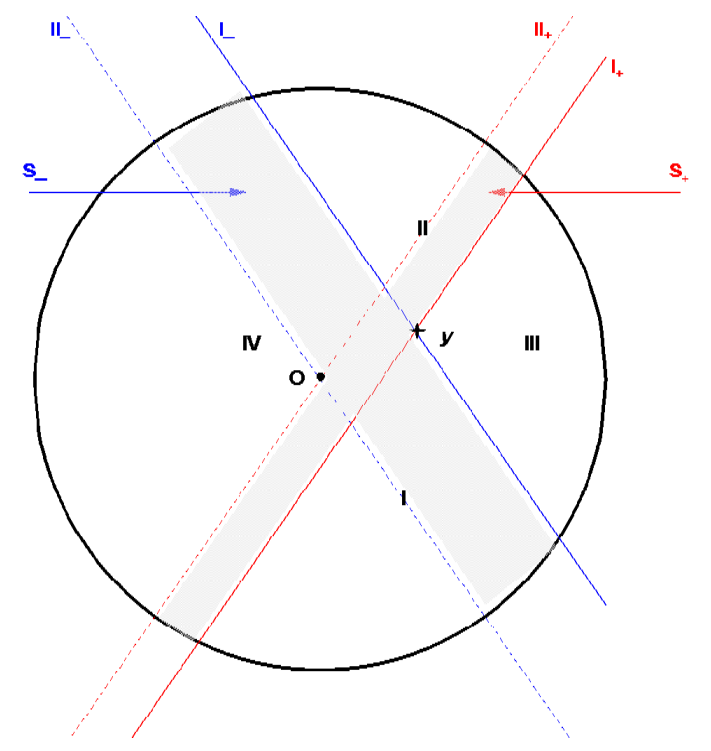

Figure 2: Illustration of the different regions needed for determining the area of the winning set for the interest group model. The point $y$ corresponds to the incumbent's position. The lines $l_{-}$and $l_{+}$through $y$ divide the issue space in four regions: regions $I$ and $I I$ (below and above $y$ respectively, containing ideal positions of voters joining interest groups on the second issue, see Lemma 3 ) and the regions $I I I$ and $I V$ (to the right and to the left of $y$, respectively, containing the ideal positions of voters joining interest groups on the first issue). The shaded region between $l_{-}$and $l l_{-}$(between $l_{+}$ and $\left.l l_{+}\right)$corresponds to $S_{-}(y ; s)\left(S_{+}(y ; s)\right)$.

and $y_{2} \geq 0$. In order to determine the sizes of the interest groups take the two lines $l_{-}$and $l_{+}$, which go through $y$ with slopes $-\sqrt{\frac{s_{1}}{s_{2}}}$ and $\sqrt{\frac{s_{1}}{s_{2}}}$, respectively. These two lines demarcate four regions in the issue space (see Figure 2), which we denote as $I$ (below $y$ ), $I I$ (above $y$ ), III (to the right of $y$ ) and $I V$ (to the left of $y$ ). For each of the regions it is easy to determine whether voters with an ideal point in that region (and with weight vector $s$ ) will join an interest group on the first issue or one on the second issue.

Lemma 3 Consider voter $j$ with ideal point $x^{j}$ and weight vector $s$.

i) If $x^{j} \in I \cup I I$ then voter $j$ derives the highest utility from the group on the second issue.

ii) If $x^{j} \in I I I \cup I V$ then voter $j$ derives the highest utility from the group on the first issue.

Figure 2 also illustrates the lines $l l_{-}$and $l l_{+}$, which pass through the origin $O$ and lie parallel to $l_{-}$and $l_{+}$, and the regions $S_{+}(y ; s)$ and $S_{-}(y ; s)$ which lie between $l_{+}$ and $l l_{+}$and between $l_{-}$and $l l_{-}$, respectively. The significance of these two regions will become evident shortly. 

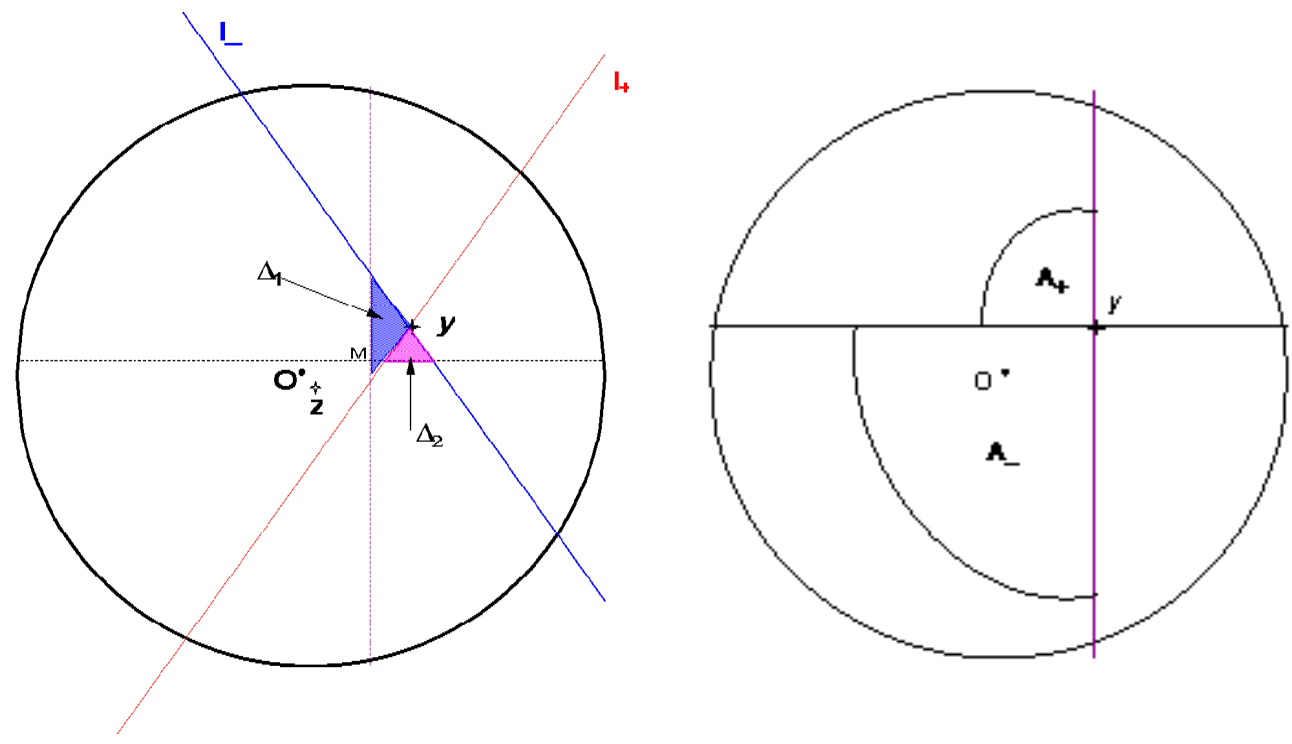

Figure 3: Illustration of Proposition 4. The left panel shows how the set $A_{-}(y ; s)$ can be constructed, the right panel show how the sets $A_{-}(y ; s)$ and $A_{+}(y ; s)$ look like.

We are now ready to prove our main results. First, in Proposition 4 we characterize, given the incumbent's position $y \neq 0$ and a weight vector $s$, the set of positions for the challenger that attract more than half of the voters with that weight vector. Proposition 5 then looks at the intersection of all these sets over different weight vectors, in order to find a lower bound for $\left|W^{I}(y)\right|$. Finally, comparing this lower bound with the upper bound for $|W(y)|$ that was found in Proposition 2, Theorem 1 is proven.

Proposition 4 In the presence of interest groups any element of the set $A(y ; s)$ is supported by more than half of the voters with weight vector $s$, where $A(y ; s)=$ $A_{-}(y ; s) \cup A_{+}(y ; s)$ and

$$
\begin{aligned}
& A_{-}(y ; s)=\left\{z: z_{1}<y_{1}, z_{2}<y_{2}\right\} \cap \mathcal{E}_{s}\left(y, w_{-}\right) \cap \mathcal{X}, \\
& A_{+}(y ; s)=\left\{z: z_{1}<y_{1}, z_{2} \geq y_{2}\right\} \cap \mathcal{E}_{s}\left(y, w_{+}\right) \cap \mathcal{X} .
\end{aligned}
$$

where $w_{*}, * \in\{-,+\}$ satisfies $\left\|y-w_{*}\right\|_{s}^{2}=4 \sqrt{s_{1} s_{2}}\left|S_{*}(y ; s)\right|$.

This proposition tells us that the area of $S_{-}(y ; s)$ (or $S_{+}(y ; s)$ ) defines a region $A_{-}(y ; s)$ (or $A_{+}(y ; s)$ ), which is part of an ellipse and has the property that all positions within this region defeat the incumbent for the given weight profile. The proof of this proposition can be illustrated by the left panel in Figure 3. Pick an arbitrary $z<y$ and denote by $m$ the midpoint of the line connecting $z$ with the incumbent's position $y$. Now draw a vertical and a horizontal line through $m$. The intersections of these lines with $l_{+}$and $l_{-}$define two triangles, $\triangle_{1}$ and $\triangle_{2}$. By construction (see Lemma 3) all voters with weight profile $s$ and an ideal point below $l_{-}$will vote for $z$, except those voters with ideal points in one of the two triangles. 
Hence we have to determine for which positions $z$ the area of the associated triangles is not too large. In fact, from Figure 2 it follows that the area below $l_{-}$can be described as $\left|l_{-}(O)\right|=\frac{1}{2}|\mathcal{X}|+\left|S_{-}(y ; s)\right|$. Moreover, the area corresponding to the voters that cast their vote for the incumbent is given by $|\mathcal{X}|-\left(\left|l_{-}(O)\right|-\left|\triangle_{1}+\triangle_{2}\right|\right)$. Using the expression for $\left|l_{-}(O)\right|$ we find that the challenger wins the election when $\left|\triangle_{1}+\triangle_{2}\right|<\left|S_{-}(y ; s)\right|$. This inequality holds exactly for all $z \in A_{-}(y ; s)$. A similar argument can be made for $A_{+}(y ; s)$. The sets $A_{-}(y ; s)$ and $A_{+}(y ; s)$ are illustrated in the right panel of Figure 3.

The set $A(y ; s)$ can be constructed for any weight profile $s$. Clearly, a policy position $z$ which lies in the intersection $\cap_{s} A(y ; s)$ of these sets has the property that for each possible weight profile a majority of the voters with this profile will vote for that policy position $z$. Any element of this set will therefore belong to the winning set $W^{I}(y)$ and the area of this intersection then gives a lower bound for $\left|W^{I}(y)\right|$. The next proposition deals with this intersection and a lower bound for its area, for the case with $y_{1}>0$ and $y_{2}>0$. For the other configurations of $y$ a similar result can be obtained.

Proposition 5 Consider $y_{1}>0$ and $y_{2}>0$. Let $R_{y}=2 \sqrt{\underline{S}(y) \sqrt{\underline{s}}}$, where $\underline{S}(y)=$ $\inf \left\{\left|S_{-}(y ; s)\right|: s_{1}, s_{2} \in \mathcal{S}\right\}$.

1. $B=B\left(y, R_{y}\right) \cap\left\{z \in \mathcal{X}: z_{i}<y_{i}, i=1,2\right\} \subset \cap_{s} A(y ; s)$;

2. $\underline{S}(y) \geq\left(K+\sqrt{K^{2}-\|y\|^{2}}\right) \frac{\left(\left|y_{1}\right|+\left|y_{2}\right|\right)}{\sqrt{\varphi(\underline{s})}}$.

We now have determined an upper bound for the number of winning positions in the absence of interest groups (Proposition 2) and a lower bound for the number of winning positions in the presence of interest groups (Proposition 5). Our main theorem then simply follows from comparing these two bounds.

Sketch of the proof of Theorem 1. From Proposition 2, part 3 we find that

$$
|W(y)| \leq \pi\|y\|^{2} \varphi(\underline{s}) \sqrt{s} .
$$

Moreover, Proposition 5, implicitly gives a lower bound for $\left|W^{I}(y)\right|$. Comparing the two, we find that a sufficient condition for $\left|W^{I}(y)\right|>|W(y)|$ is that $y$ belongs to $B(O, \Psi(\underline{s}) K) \backslash\{O\}$, where

$$
\Psi(\underline{s})=\min \left\{\frac{2(\varphi(\underline{s}))^{\frac{3}{2}}}{1+(\varphi(\underline{s}))^{3}}, \frac{1}{\sqrt{1+4 \varphi(\underline{s}) \sqrt{\underline{s}}}}\right\} .
$$

\section{Concluding remarks}

In this short note we have shown that the introduction of interest groups into a spatial voting model of electoral competition between two political parties will typically 
increase the winning set for the challenger. Since in mass elections candidates or political parties will in general lack information concerning the distribution of voter preferences, it can be quite difficult to find winning positions. This paper suggests that winning sets for the challenger will increase in the presence of interest groups, thereby increasing the probability of winning for the challenger and making it harder to locate an equilibrium point if it exists. This effect, which is driven by the intuitive assumption that interest group membership is positively related to the discontent with the incumbent's policy on the relevant issue has, to our knowledge, never been shown before. The effect is confirmed in a simulation study by Sadiraj, Tuinstra and van Winden (2004). Moreover, this simulation study suggests that, because of the increase in the winning set in the presence of interest groups, separation between policy platforms increases and the rate of convergence of policy platforms to the equilibrium point decreases.

The model we have chosen to present our main result is, of course, highly stylized. Two final remarks are in order here. First, as can be seen from Section 3, we have been quite conservative in providing upper and lower bounds of the relevant areas. We therefore expect that our main result holds for a significantly larger set than the one specified by Theorem 1. Secondly, for matters of exposition we chose as issue space a ball around the origin, where a rectangular issue space is more common in the literature. Actually, the issue space might be larger than this ball. A distribution of voter preferences over for example a square or rectangle would substantively give the same results, but would lead to a cumbersome written proof. The present assumption is also motivated by the observation that voters typically do not take extreme positions on all issues. What the precise effect of interest groups will be under a more general specification of the (dimension of the) issue space, the distribution of ideal points and the structure of voter preferences or in the absence of an equilibrium point is left for future research. Note however that the result presented here holds for any distribution of strengths, provided that this distribution is independent of the ideal points. The main message here is that interest groups will have an impact on voting behavior and thereby influence the political decision making process, even apart from their influence on the latter through lobbying, campaign contributions or endorsements, which are the channels studied in the existing political economic literature on interest groups.

\section{References}

[1] Akerlof, G.A. and R.E. Kranton (2000): Economics and identity. Quarterly Journal of Economics 115, 715-753.

[2] Austen-Smith, D. (1994): Interest groups: money, information and influence. In: Perspectives on Public Choice, D.C. Mueller (ed.), Cambridge: Cambridge University Press.

[3] Brewer, M.B. (1979), In-group bias in the minimal intergroup situation: a cognitive-motivational analysis, Psychological Bulletin 86, 307-324. 
[4] Chong, D. (1991): Collective Action and the Civil Rights Movement. Chicago: University of Chicago Press.

[5] Enelow, J.M. and M.J. Hinich (1984): The Spatial Theory of Voting: An Introduction. Cambridge: Cambridge University Press.

[6] Festinger, L. and E. Aronson (1968): Arousal and reduction of dissonance in social contexts. In: Group Dynamics, D. Cartwright and A. Zander (eds.), New York: Harper \& Row.

[7] Grossman, G.M. and E. Helpman (2001): Special Interest Politics. Cambridge: The MIT Press.

[8] Hoyer, R.W. and L.S. Mayer (1974): Comparing strategies in a spatial model of electoral competition. American Journal of Political Science 18, 501-523.

[9] Javeline, D. (2003): The role of blame in collective action: evidence from Russia. American Political Science Review 97, 107-121.

[10] Kernell, S. (1977): Presidential popularity and negative voting. American Political Science Review 71, 44-66.

[11] Kollman, K., J.H. Miller and S.E. Page (1992): Adaptive parties in spatial elections. American Political Science Review 86, 929-937.

[12] Kramer, G.H. (1977): A dynamic model of political equilibrium. Journal of Economic Theory 16, 310-334.

[13] Lau, R.R. (1982): Negativity in political perception. Political Behavior 4, 353378.

[14] Plott, C.R. (1967): A notion of equilibrium and its possibility under majority rule. American Economic Review 57, 787-806.

[15] Potters, J., and R. Sloof (1996): Interest groups: a survey of empirical models that try to assess their influence. European Journal of Political Economy 12, 403-442.

[16] Richardson, J.J. (ed.) (1994): Pressure Groups. New York: Oxford University Press.

[17] Romer, P. (1996): Preferences, promises, and the politics of entitlement. In: Individual Social Responsibility, V. Fuchs (ed.), Chicago: University of Chicago Press.

[18] Sadiraj, V., J. Tuinstra and F. van Winden (2004): A computational electoral competition model with social clustering and endogenous interest groups as information brokers. CeNDEF Working paper 04-08, University of Amsterdam.

[19] Sadiraj, V., J. Tuinstra and F. van Winden (2005): Interest group size dynamics and policymaking. Forthcoming in: Public Choice. 
[20] Tajfel, H. and J.C. Turner (1986): The social identity theory of intergroup behavior. In: S. Worchel and W.G. Austin (Eds.), Psychology of Intergroup Relations, Chicago: Nelson-Hall.

[21] van Winden, F. (2003): Interest group behavior and influence. In C.K. Rowley and F. Schneider (eds.), Encyclopedia of Public Choice. Boston: Kluwer Academic Publishers.

\section{A Proofs}

This appendix contains formal proofs for the results in Section 3.

\section{Proof of Proposition 2.}

1. By symmetry, any line through the origin $O$ divides the issue space $\mathcal{X}$ in two subspaces which are equally large. The uniform distribution then implies the same expected number of voters on either side of such a line. Therefore the origin corresponds to the position of the generalized median voter and no position will strictly defeat it. Hence $W(O)=\emptyset$.

2. Without loss of generality we assume $y_{1} \geq y_{2}>0$. Consider an arbitrary policy position $z$, with $z_{i} \leq y_{i}, i=1,2$. We want to determine under which conditions $z \in W(y)$. Let us first determine all positions $x^{*}$ such that voters with the considered weight profile $s$ are indifferent between $z$ and $y$. These positions $x^{*}$ have to satisfy

$$
\left\|y-x^{*}\right\|_{s}^{2}=\left\|z-x^{*}\right\|_{s}^{2} .
$$

Solving for $x_{2}^{*}$ is straightforward and gives

$$
x_{2}^{*}=\frac{-2 s_{1}\left(y_{1}-z_{1}\right) x_{1}^{*}+\|y\|_{s}^{2}-\|z\|_{s}^{2}}{2 s_{2}\left(y_{2}-z_{2}\right)} .
$$

Let us denote the line defined by (3) as $l_{s}^{*}$. Clearly, $l_{s}^{*}$ separates the issue space into two subspaces and all voters in the subspace below and to the left of $l_{s}^{*}$ vote for the challenger, if it selects position $z$. Now suppose $l_{s}^{*}$ cuts the vertical axis at some positive value. Then we can draw a line that goes through $O$ and that lies parallel to $l_{s}^{*}$. This line through $O$ divides $\mathcal{X}$ in two equal subspaces, implying that the subspace lying below and to the left of $l_{s}^{*}$ will be larger than the subspace above and to the right of $l_{s}^{*}$. Therefore, the challenger will win at $z$ if $l_{s}^{*}$ intersects the vertical axis at some positive value. This condition reduces to

$$
\|y\|_{s}^{2}-\|z\|_{s}^{2}>0
$$

which defines the ellipse given in the proposition. In a similar fashion the same condition can be derived for positions $z$ with $z_{1} \leq y_{1}$ and $z_{2}>y_{2}$ and for positions $z$ with $z_{1}>y_{1}$ and $z_{2} \leq y_{2}$. It should be clear that positions $z$ with $z_{1} \geq y_{1}$ and $z_{2} \geq y_{2}$ never defeat the incumbent. 
3. First, we show that $W(y) \subset \bigcup_{c \in C} \mathcal{E}_{c}(O, y)$. A sufficient condition for this to be true is $\cup_{s} \mathcal{E}_{s}(O, y) \subset \cup_{c} \mathcal{E}_{c}(O, y)$.

Let $x \in \cup_{s} \mathcal{E}_{s}(O, y)$. Hence, $x \in \mathcal{E}_{s}(O, y)$ for some $s_{1}, s_{2} \in \mathcal{S}$ and therefore $\|x\|_{s}^{2}<\|y\|_{s}^{2}$, or equivalently

$$
\left(x_{1}^{2}-y_{1}^{2}\right)<\frac{s_{2}}{s_{1}}\left(y_{2}^{2}-x_{2}^{2}\right) .
$$

Note that $\underline{s} \leq \frac{s_{2}}{s_{1}} \leq \frac{1}{s}$, for all $s_{1}, s_{2} \in \mathcal{S}$. If $\left|x_{2}\right| \leq\left|y_{2}\right|$, then

$$
\left(x_{1}^{2}-y_{1}^{2}\right)<\frac{s_{2}}{s_{1}}\left(y_{2}^{2}-x_{2}^{2}\right) \leq \frac{1}{\underline{s}}\left(y_{2}^{2}-x_{2}^{2}\right) \Longleftrightarrow \underline{s} x_{1}^{2}+x_{2}^{2} \leq \underline{s} y_{1}^{2}+y_{2}^{2}
$$

and therefore $x \in \mathcal{E}_{c}(O, y)$ with $c=(\underline{s}, 1)$. On the other hand, if $\left|x_{2}\right|>\left|y_{2}\right|$ we have

$$
\left(x_{1}^{2}-y_{1}^{2}\right)<\frac{s_{2}}{s_{1}}\left(y_{2}^{2}-x_{2}^{2}\right) \leq \underline{s}\left(y_{2}^{2}-x_{2}^{2}\right),
$$

and therefore $x \in \mathcal{E}_{c}(O, y)$ with $c=(1, \underline{s})$.

Second, we calculate the area of $\bigcup_{c \in C} \mathcal{E}_{c}(O, y)$. Recall that the surface of $\mathcal{E}_{c}(O, y)$ is given by $\pi \frac{\|y\|_{c}^{2}}{\sqrt{c_{1} c_{2}}}$ and therefore we have

$$
\begin{aligned}
\left|\bigcup_{c \in C} \mathcal{E}_{c}(O, y)\right| & \leq \sum_{c \in C} \pi \frac{\|y\|_{c}^{2}}{\sqrt{c_{1} c_{2}}} \\
& =\frac{\pi}{\sqrt{\underline{s}}}\left(\|y\|_{(\underline{s}, 1)}^{2}+\|y\|_{(1, \underline{s})}^{2}\right) \\
& =\pi \varphi(\underline{s}) \sqrt{s}\|y\|^{2}
\end{aligned}
$$

Hence,

$$
|W(y)| \leq\left|\bigcup_{c \in C} \mathcal{E}_{c}(O, y)\right| \leq \pi \varphi(\underline{s}) \sqrt{\underline{s}}\|y\|^{2} .
$$

We need the following definitions (see Figures 2 and 3). Let $\bullet$ denote the scalar product operator, i.e. $u \bullet v=\sum_{i} u_{i} v_{i}$, and let $l_{u, v}$ denote the line through $v$ and perpendicular to $u$, i.e. $l_{u, v}=\{x: u \bullet(x-v)=0\}$.

Definition 6 Define $l_{+}$and $l_{-}$as follows: $l_{+}=l_{\left(-\sqrt{s_{1}}, \sqrt{s_{2}}\right), y}$ and $l_{-}=l_{\left(\sqrt{s_{1}}, \sqrt{s_{2}}\right), y}$. These lines divide the issue space $\mathcal{X}=B(O, K)$ in the following four subspaces.

1. $I=\left\{x \in \mathcal{X}: x_{2}<\min \left\{l_{-}, l_{+}\right\}\right\}$,

2. $I I=\left\{x \in \mathcal{X}: x_{2}>\max \left\{l_{-}, l_{+}\right\}\right\}$ 
3. $I I I=\left\{x \in \mathcal{X} \backslash\left(I \cup I I \cup l_{-} \cup l_{+}\right): x_{1}>y_{1}\right\}$ and

4. $I V=\left\{x \in \mathcal{X} \backslash\left(I \cup I I \cup l_{-} \cup l_{+}\right): x_{1}<y_{1}\right\}$

Clearly, $\mathcal{X}=I \cup I I \cup I I I \cup I V \cup l_{-} \cup l_{+}$

Denote by $l l_{-}$and $l l_{+}$the lines passing through the origin $O$, and parallel to $l_{-}$ and $l_{+}$, respectively. That is $l l_{+}=l_{\left(-\sqrt{s_{1}}, \sqrt{s_{2}}\right), O}$ and $l l_{-}=l_{\left(\sqrt{s_{1}}, \sqrt{s_{2}}\right), O \text {. Let }}$

$$
\begin{aligned}
& S_{+}(y ; s)=\left\{x \in \mathbb{R}^{2} \mid \min \left(l l_{+}, l_{+}\right) \leq x_{2} \leq \max \left(l l_{+}, l_{+}\right)\right\} \cap \mathcal{X}, \\
& S_{-}(y ; s)=\left\{x \in \mathbb{R}^{2} \mid \min \left(l l_{-}, l_{-}\right) \leq x_{2} \leq \max \left(l l_{-}, l_{-}\right)\right\} \cap \mathcal{X} .
\end{aligned}
$$

Using $S_{+}(y ; s)$ and $S_{-}(y ; s)$ we define

$$
\begin{aligned}
& A_{-}(y ; s)=\left\{z: z_{1}<y_{1}, z_{2}<y_{2}\right\} \cap \mathcal{E}_{s}\left(y, w_{-}\right) \cap \mathcal{X} \\
& A_{+}(y ; s)=\left\{z: z_{1}<y_{1}, z_{2} \geq y_{2}\right\} \cap \mathcal{E}_{s}\left(y, w_{+}\right) \cap \mathcal{X} .
\end{aligned}
$$

where $w_{*}, * \in\{-,+\}$ satisfies $\left\|y-w_{*}\right\|_{s}^{2}=4 \sqrt{s_{1} s_{2}}\left|S_{*}(s, y)\right|$. Finally $A(y ; s)=$ $A_{-}(y ; s) \cup A_{+}(y ; s)$.

Proof of Lemma 3. We will show that if a voter has an ideal position in $I$ then she would prefer the group on the second issue to the group on the first one. The result for voters from subspaces $I I, I I I$ and $I V$ can be established in the same way. Consider voter $j$ with ideal position $x^{j} \in I$. Note that $\min \left\{l_{-}, l_{+}\right\}<y_{2}$ implies that

$$
\left|x_{2}^{j}-y_{2}\right|=-\left(x_{2}^{j}-y_{2}\right) \text {. }
$$

We distinguish the following cases:

- $x_{1}^{j} \leq y_{1}$. We then have

(a) $\left|x_{1}^{j}-y_{1}\right|=y_{1}-x_{1}^{j}$, and

(b) $\min \left\{l_{+}, l_{-}\right\}=l_{+}$. Therefore $x_{2}^{j}<y_{2}+\sqrt{\frac{s_{1}}{s_{2}}}\left(x_{1}^{j}-y_{1}\right)$ and hence

$$
-\left(x_{2}^{j}-y_{2}\right)>\sqrt{\frac{s_{1}}{s_{2}}}\left(y_{1}-x_{1}^{j}\right) .
$$

Substituting (a) and (4) at the above inequality,

$$
\sqrt{s_{2}}\left|x_{2}^{j}-y_{2}\right|>\sqrt{s_{1}}\left|x_{1}^{j}-y_{1}\right|
$$

which is a necessary and sufficient condition for voter $j$ to prefer the interest group on the second issue over the interest group on the first issue.

- $x_{1}^{j}>y_{1}$. Note that,

(c) $\left|x_{1}^{j}-y_{1}\right|=x_{1}^{j}-y_{1}$, 
(d) $\min \left\{l_{+}, l_{-}\right\}=l_{-}$, and by a similar argument as above one obtains

$$
\sqrt{s_{2}}\left|x_{2}^{j}-y_{2}\right|>\sqrt{s_{1}}\left|x_{1}^{j}-y_{1}\right|
$$

which is a necessary and sufficient condition for a voter $j$ to prefer the group on the second issue $x_{1}^{j}=y_{1}$.

Proof of Proposition 4. Consider a position $z \in A_{-}(y ; s)$. Denote by $M=$ $\left(\frac{y_{1}+z_{1}}{2}, \frac{y_{2}+z_{2}}{2}\right)$ the midpoint of the line connecting $z$ with the incumbent's position $y$. Now draw a vertical and a horizontal line through $m$ and consider the intersections of these lines with $l_{+}$and $l_{-}$. Denote these intersections by $i_{*}$, where $* \in\{+,-\}$ and $i \in\{1,2\}$. Hence $1_{+}\left(1_{-}\right)$is the intersection between the vertical line through $M_{1}$ and $l_{+}\left(l_{-}\right)$and $2_{+}\left(2_{-}\right)$is the intersection between the horizontal line through $M_{2}$ and $l_{+}\left(l_{-}\right)$. Now consider the subspace $\mathcal{L} \equiv\left(l_{-}(O)-\left(1_{-} 1_{+} 2_{+} 2_{-} 1_{-}\right)\right) \cap \mathcal{X}$. From Lemma 3 we know that all voters in $I$ join the interest group on the second issue and all voters in $I V$ join the interest group on the first issue. For the voters in $I \cap \mathcal{L}$ we have $\left|x_{2}^{j}-z_{2}\right|<\left|x_{2}^{j}-y_{2}\right|$ and for all voters in $I V \cap \mathcal{L}$ we have $\left|x_{1}^{j}-z_{1}\right|<\left|x_{1}^{j}-y_{1}\right|$. This proves that the challenger at position $z$ gets votes from voters with ideal positions in $\mathcal{L}$. Hence it gets more than half of the votes if

$$
\left|S_{-}\right|>\left|1_{-} 1_{+} 2_{+} 2_{-} 1_{-}\right|
$$

since $\left|S_{-}\right|=\left|l_{-}(O)\right|-\frac{1}{2}|\mathcal{X}|$.

First, denote $\triangle_{1}$ the triangle $y 1_{+} 1_{-}$and $\triangle_{2}$ the triangle $y 2_{+} 2_{-}$and note that

$$
\begin{aligned}
\left|\left(1_{-} 1_{+} 2_{+} 2_{-} 1_{-}\right) \cap \mathcal{X}\right| & \leq\left|\triangle_{1}\right|+\left|\triangle_{2}\right| \\
& =\sqrt{\frac{s_{1}}{s_{2}}}\left(y_{1}-M_{1}\right)^{2}+\sqrt{\frac{s_{2}}{s_{1}}}\left(y_{2}-M_{2}\right)^{2} \\
& =\frac{1}{4} \sqrt{\frac{s_{1}}{s_{2}}}\left(y_{1}-z_{1}\right)^{2}+\frac{1}{4} \sqrt{\frac{s_{2}}{s_{1}}}\left(y_{2}-z_{2}\right)^{2} \\
& \leq\left|S_{-}\right|
\end{aligned}
$$

The first inequality follows from the fact that some parts of the triangles $\triangle_{i}, i=1,2$ might not be in $\mathcal{X}$, the first equal sign follows from computation of the surface of the two triangles, the second equality follows from the definition of the midpoint $M$ and the final step follows from the fact that $z \in A_{-}(y ; s)$. A similar reasoning holds for $A_{+}(y ; s)$. Thus, it is shown that all positions $z \in A(y ; s)$ are expected to defeat the incumbent.

\section{Proof of Proposition 5.}

1. First, we show that

$$
B \subset \cap_{s} A(y ; s)
$$

Indeed, let $x \in B$ i.e. $x_{i}<y_{i}$ and

$$
\|x-y\|^{2} \leq R_{y}^{2}=4 \underline{S} \sqrt{\underline{s}}
$$


Thus, $\forall s \in \mathcal{S}^{2}$ and $c=\left(\frac{1}{4} \sqrt{\frac{s_{1}}{s_{2}}}, \frac{1}{4} \sqrt{\frac{s_{2}}{s_{1}}}\right)$

$$
\begin{aligned}
\|x-y\|_{c}^{2} & \leq \frac{1}{4} \sqrt{\frac{1}{\underline{s}}}\|x-y\|^{2} \\
& =\frac{1}{4} \sqrt{\frac{1}{\underline{s}}} 4 \underline{S} \sqrt{\underline{s}} \\
& \leq\left|S_{-}(y ; s)\right|
\end{aligned}
$$

and therefore, $x \in A_{-}(y ; s) \subset A(y ; s)$.

2. The next step consists of deriving a lower bound for the radius of $B$. First, let $d\left(O, l_{+}\right)\left(d\left(O, l_{-}\right)\right)$correspond to the distance of the origin from the line $l_{+}$ $\left(l_{-}\right)$, i.e.

$$
d\left(O, l_{+}\right)=\min \left\{\|x\|: x \in l_{+}\right\} .
$$

For the case we are considering $\left(y_{1}>0\right.$ and $\left.y_{2}>0\right)$, we have $h(y ; s)=d\left(O, l_{-}\right)$ (which means that we are focussing on $A_{-}(y ; s)$ ). For all $s \in \mathcal{S}^{2}$, one has

$$
\begin{aligned}
\left|S_{-}(y, s)\right| & =2 \int_{0}^{h(y ; s)} \sqrt{K^{2}-z^{2}} d z \\
& =\left[z \sqrt{K^{2}-z^{2}}+K^{2} \arcsin \frac{z}{K}\right]_{0}^{h(y ; s)} \\
& =h(y ; s) \sqrt{K^{2}-h(y ; s)^{2}}+K^{2} \arcsin \frac{h(y ; s)}{K} .
\end{aligned}
$$

By definition we have $h(y ; s) \leq\|y\| \leq K$. Furthermore, $\arcsin x>x$ for all $x \in(0,1]$. Using these properties we find

$$
\left|S_{-}(y, s)\right| \geq \underline{h}(y) \sqrt{K^{2}-\|y\|^{2}}+K,
$$

where $\underline{h}(y)=\inf _{s_{1}, s_{2} \in \mathcal{S}} h(y ; s)$. Finally, since $\underline{s} \leq \min \left\{s_{1} / s_{2}, s_{2} / s_{1}\right\}$ and therefore $\frac{\sqrt{\underline{s}}}{\sqrt{1+\underline{s}}} \leq \min \left\{\frac{\sqrt{s_{1}}}{\sqrt{s_{1}+s_{2}}}, \frac{\sqrt{s_{2}}}{\sqrt{s_{1}+s_{2}}}\right\}$, we have

$$
\begin{aligned}
h(y ; s) & =\frac{\sqrt{s_{1}}\left|y_{1}\right|+\sqrt{s_{2}}\left|y_{2}\right|}{\sqrt{s_{1}+s_{2}}} \\
& \geq \frac{\sqrt{\underline{s}}}{\sqrt{1+\underline{s}}}\left(\left|y_{1}\right|+\left|y_{2}\right|\right)
\end{aligned}
$$

which implies $\underline{h}(y) \geq \frac{\sqrt{\underline{s}}\left(\left|y_{1}\right|+\left|y_{2}\right|\right)}{\sqrt{1+\underline{s}}}$. Using $\varphi(s)=1+1 / s$ one now has

$$
\left|S_{-}(y ; s)\right| \geq \sqrt{1 / \varphi(\underline{s})}\left(\sqrt{K^{2}-\|y\|^{2}}+K\right)\left(\left|y_{1}\right|+\left|y_{2}\right|\right)
$$


Proof of Theorem 1. Let $\underline{s}$ be given. From proposition 2 we know that

$$
|W(y)| \leq \pi\|y\|^{2} \sqrt{s} \varphi(s) .
$$

From Proposition 5 we know that all positions $z$ in $B(y, 2 \sqrt{\sqrt{\underline{s}}}|\underline{S}|)$, with $z_{1}<y_{1}$ and $z_{2}<y_{2}$ are contained in $W^{I}(y)$. Furthermore we found that

$$
\left|S_{-}(y, s)\right| \geq \underline{S}(y) \geq \sqrt{1 / \varphi(\underline{s})}\left(K+\sqrt{K^{2}-\|y\|^{2}}\right)\left(\left|y_{1}\right|+\left|y_{2}\right|\right) .
$$

If this area lies in $\mathcal{X}$ we know that it presents a lower bound for $\left|W^{I}(y)\right|$. This does not necessarily have to be the case. Let us first compute the two points that have one of the coordinates equivalent with the point $y$ but that lie on the border of $\mathcal{X}$. These points are $\left(y_{1}, \sqrt{K^{2}-y_{1}^{2}}\right)$ and $\left(\sqrt{K^{2}-y_{2}^{2}}, y_{2}\right)$. Now consider the following ball $B(y, r)$ where $r=\min \left\{\left|y_{1}\right|+\sqrt{K^{2}-y_{2}^{2}},\left|y_{2}\right|+\sqrt{K^{2}-y_{1}^{2}}\right\}$. One fourth of this ball lies in $\mathcal{X}$ completely. Therefore there are two possibilities. This fourth part of the ball is contained in $A_{-}$or it contains $A_{-}$. So we have to take the minimum of the two lower bounds as a lower bound for $\left|W^{I}(y)\right|$. Consider the first case. Then

$$
\left|W^{I}(y)\right| \geq \pi \sqrt{\underline{s} / \varphi(\underline{s})}\left(K+\sqrt{K^{2}-\|y\|^{2}}\right)\left(\left|y_{1}\right|+\left|y_{2}\right|\right)
$$

and

$$
|W(y)|<\pi\|y\|^{2} \sqrt{s} \varphi(\underline{s})
$$

So we find that, in the presence of interest groups, the size of the winning set is expected to increase for all $y$ satisfying $\left|W^{I}(y)\right| \geq|W(y)|$ or

$$
\pi \sqrt{\underline{s} / \varphi(\underline{s})}\left(K+\sqrt{K^{2}-\|y\|^{2}}\right)\left(\left|y_{1}\right|+\left|y_{2}\right|\right) \geqslant \pi\|y\|^{2} \sqrt{\underline{s}} \varphi(\underline{s})
$$

Using $\left|y_{1}\right|+\left|y_{2}\right| \geq\|y\|$, and rewriting we find that for all $y$ satisfying

$$
\|y\| \leq \frac{2(\varphi(\underline{s}))^{\frac{3}{2}}}{1+(\varphi(\underline{s}))^{3}} K
$$

our property holds. Defining $D_{1} \equiv \frac{2(\varphi(\underline{s}))^{\frac{3}{2}}}{1+(\varphi(\underline{s}))^{3}} K$, the property holds for all $y \in B\left(0, D_{1}\right)$.

Now consider the second case with

$$
\left|W^{I}(y)\right| \geq \frac{\pi}{4}\left(\min \left\{\left|y_{1}\right|+\sqrt{K^{2}-y_{2}^{2}},\left|y_{2}\right|+\sqrt{K^{2}-y_{1}^{2}}\right\}\right)^{2} .
$$

Suppose, without loss of generality, that $\left|y_{1}\right|+\sqrt{K^{2}-y_{2}^{2}} \leq\left|y_{2}\right|+\sqrt{K^{2}-y_{1}^{2}}$. We then obtain

$$
\frac{\pi}{4}\left(\left|y_{1}\right|+\sqrt{K^{2}-y_{2}^{2}}\right)^{2} \geq \pi\|y\|^{2} \sqrt{s} \varphi(\underline{s}) .
$$


Again, we will try to derive a condition on $\|y\|^{2}$. Using $0 \leq\left|y_{2}\right|^{2} \leq\|y\|^{2}$, we get

$$
\left|y_{1}\right|^{2}+K^{2}-y_{2}^{2}+2\left|y_{1}\right| \sqrt{K^{2}-y_{2}^{2}} \geq K^{2}-\|y\|^{2} \geq 4\|y\|^{2} \varphi(\underline{s}) \sqrt{\underline{s}}
$$

or

$$
\|y\|^{2} \leq \frac{K^{2}}{1+4 \varphi(\underline{s}) \sqrt{\underline{s}}}
$$

That is, for all $y \in B\left(0, D_{2}\right)$, with $D_{2} \equiv \frac{K}{\sqrt{1+4 \varphi(s) \sqrt{\underline{s}}}}$. This concludes the proof of Theorem 1. 